\title{
Perfil sociodemográfico del aborto inducido
}

Evelio Cabezas-García, M.C ., M. en C.,1) A na Langer-G lass, M.C., ${ }^{(2)}$

Luisa Alvarez-Vázquez, Dr. en C., ${ }^{(3)}$ Patricia Bustamante, M. en C. ${ }^{(4)}$

\section{Cabezas-García E, Langer-Glass A, Alvarez-Vázquez $L$, Bustamante $P$. Perfil sociodemográfico del aborto inducido. Salud Publica Mex 1998;40:265-271.}

\section{Resumen}

Objetivo. Identificar características sociodemográficas y su fuerza de asociación con el aborto inducido del primer embarazo. Material y métodos. Se realizó un estudio analítico con la información de una encuesta realizada en el municipio Diez de 0 ctubre, de la ciudad de La Habana, en Cuba, durante todo el año de 1991 y el primer semestre de 1992. La población de estudio se dividió en dos grupos de comparación: uno formado por las mujeres cuyo primer embarazo terminó en un aborto inducido y el otro constituido por aquellas cuyo embarazo llegó a término. De las variables estudiadas que mostraron diferencias estadísticamente significativas se obtuvieron las razones de momios crudas y ajustadas por la variable que se consideró como confusora: la edad. Para esta última fase del análisis se utilizó la regresión logística multivariada. Resultados. Las características socio demográficas identificadas como factores de riesgo asociados al aborto inducido del primer embarazo fueron la edad menor de 24 años (aunque el riesgo se incrementa en las menores de 20 años) y ser solteras 0 unidas. Conclusiones. El riesgo de recurrir a la práctica del aborto inducido en el primer embarazo es elevado en mujeres muy jóvenes que aún no han cumplido sus expectativas profesionales, laborales y relacionadas con el matrimonio. Estas razones parecen incompatibles con la maternidad en el grupo de mujeres estudiadas.

Palabras clave: aborto inducido; Cuba

\author{
Cabezas-García E, Langer-Glass A, \\ Alvarez-Vázquez L, Bustamante P. \\ Sociodemographic profile \\ of induced abortion.
}

Salud Publica Mex 1998;40:265-271.

\begin{abstract}
A bstract
Objective. To identify sociodemographic characteristics associated with induced abortion of the first pregnancy and quantify the strength of association between them. Material and methods. D ata were gathered from a survey conducted in the district of Diez de $\mathrm{O}$ ctubre, Havana, Cuba throughout 1991 and the beginning of 1992.T he study population was divided into two comparable groups: one group of women whose first pregnancy terminated in induced abortion and a second group of women whose pregnancy terminated in childbirth. For the variables with statistically significant differences, both the crude and adjusted odds ratio were obtained for the one potentially confounding factor: age. Multivariate logistic regression analysis was employed in the final stage. Results. The sociodemographic characteristic identified as risk factor for induced abortion during the first pregnancy is being younger than 24 years of age, a risk which increased with women who were less than 20 years old, whether single or in union. Conclusions. Recurrence risk of induced abortion during the first pregnancy is higher in younger women who have not achieved their professional, working or marrying expectations. This situation seem to be incompatible with maternity in the studied group.
\end{abstract}

Key words: induced abortion; Cuba

(1) Investigador de la Dirección General de Salud Reproductiva, Secretaría de Salud, México.

(2) Directora Regional del Population Council para América Latina y el Caribe, México.

(3) Investigadora del Instituto $\mathrm{N}$ acional de Endocrinología y del Centro $\mathrm{N}$ acional de Demografía de Cuba, La Habana, Cuba.

(4) Investigadora del Instituto N acional de Salud Pública de México, México.

Fecha de recibido: 9 de septiembre de 1997 - Fecha de aprobado: 13 de marzo de 1998 Insurgentes Sur 1397, 60. piso, colonia Insurgentes Mixcoac, 03920 México, D.F., México. 
$\mathrm{L}$ a fecundidad en Cuba ha experimentado un gran descenso a partir de los años sesenta, hasta alcanzar un nivel bajo en la actualidad. Según datos del Ministerio de Salud Pública, el número de hijos se redujo notablemente de 4.7 por mujer en 1963, a 1.5 en 1992 , lo que constituye una de las tasas de fecundidad más bajas de América Latina. ${ }^{1,2}$

Los factores más importantes que han determinado el descenso de la fecundidad son la utilización de métodos anticonceptivos y la práctica del aborto.

El aborto inducido no es un fenómeno nuevo en Cuba: existen evidencias de que ya desde la colonia las esclavas recurrían a esa práctica; sin embargo, no existen cifras oficiales sobre el número de abortos que se practicaron en Cuba antes de 1968. Los datos de encuestas realizadas en los años setenta permiten tener una idea de lo ocurrido en etapas anteriores, y se ha llegado a la conclusión de que, a pesar de la existencia de leyes restrictivas en contra del aborto inducido, éste era frecuente y predominaba en zonas urbanas, sobre todo en las capitales de provincia. ${ }^{*}$

Después de la Revolución, en 1964 se enmendó el Código de Defensa Social y, sin cambiar realmente el artículo 433 sobre el aborto, los nuevos criterios permitieron que se brindaran las mejores condiciones para su realización, con el propósito de proteger la salud de la mujer. La práctica del aborto no está penalizada siempre que se cumplan las siguientes condiciones: que no sea realizado por lucro y que se lleve a cabo en las instituciones oficiales por personal médico calificado y con el consentimiento de la paciente. ${ }^{3}$

Las características del aborto inducido en Cuba han permitido reducir en $60 \%$ las muertes maternas relacionadas con este proceder quirúrgico entre los

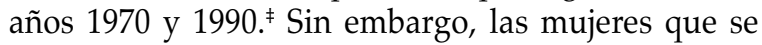
someten a esa práctica no están exentas de presentar complicaciones en el corto y en el largo plazo, desde el punto de vista tanto ginecológico como psicológico, por lo que las autoridades nacionales e internacionales reconocen que la elevada tasa de aborto inducido constituye un problema de salud para la mujer cubana. ${ }^{1}$

El mejor conocimiento de las determinantes del aborto inducido y la posibilidad de incidir sobre ellas mediante programas o políticas, permitirán reducir su magnitud, así como atenuar las consecuencias

\footnotetext{
* En los Anales de la Academia de Ciencias Físicas y Naturales, se pueden encontrar trabajos de prestigiados médicos que estudiaron este tema, como los de Núñez Palomino, Eusebio Hernández, Gabriel Casuso y Julio Ortiz.

* Cabezas E. Mortalidad materna en Cuba. Trabajo presentado en la Conferencia Centroamericana sobre Maternidad sin Riesgo; enero de 1992; Guatemala, Guatemala. Documento no publicado.
}

sobre la salud reproductiva de la mujer y el impacto que esta condición causa en los servicios hospitalarios del país.

Esta investigación se llevó a cabo con dos objetivos: identificar características sociodemográficas asociadas con el aborto inducido del primer embarazo y cuantificar la fuerza de asociación de las mismas con el aborto inducido.

\section{Material y métodos}

Se realizó un estudio analítico con la información obtenida por una encuesta sobre aborto, realizada en el municipio Diez de Octubre, el más populoso de la ciudad de La Habana, durante todo el año de 1991 y el primer semestre de 1992.

El tamaño muestral necesario para el estudio se determinó a partir de indicadores de aborto, estadísticas vitales, otras investigaciones sobre fecundidad realizadas en el municipio seleccionado y tomando en cuenta el diseño muestral. Se realizó un muestreo por conglomerado monoetápico, en el que los conglomerados fueron los consultorios del médico de la familia, y las unidades de análisis, las mujeres de 13 a 34 años, residentes en el momento de la encuesta en dicho municipio, mismas que fueron entrevistadas en sus casas.

Para la encuesta se utilizó un cuestionario estructurado con preguntas de opción múltiple (precodificado) y preguntas abiertas. El mismo fue validado previamente mediante un estudio piloto que permitió contar con un cuestionario claro y preciso para evitar sesgos de información.

Para los fines de este trabajo se seleccionaron las mujeres que estuvieron embarazadas antes de la encuesta y se excluyeron las que nunca habían estado embarazadas o que estaban embarazadas por primera vez al momento de la encuesta.

Del total de mujeres seleccionadas $(\mathrm{N}=1528)$ se formaron dos grupos de comparación: uno de mujeres cuyo primer embarazo terminó en un aborto inducido (659), y otro con aquellas cuyo primer embarazo llegó a término (869 mujeres).

Los datos se capturaron con el programa FOXBASE y se analizaron con el programa estadístico STATA.

Se obtuvieron las frecuencias relativas de las variables categóricas estudiadas para ambos grupos de comparación y se utilizó la prueba estadística de chi cuadrada, con un nivel de confianza de 95\% ( $p<0.05)$.

Posteriormente se calcularon las razones de momios (RM) crudas y sus intervalos de confianza (IC) para aborto inducido o parto, con cada una de las variables del estudio que mostraron diferencias es- 
tadísticamente significativas. Puesto que se contó con diferentes categorías de las variables independientes, se obtuvo una medida de asociación por estrato.

Debido a que el evento bajo estudio fue un factor binario (aborto inducido/ parto), se utilizó la regresión logística para la estimación de RM ajustadas por la variable confusora. Se consideró como variable potencialmente confusora la edad, y el criterio para ello fue una diferencia mayor de $10 \%$ entre la RM cruda y la RM ajustada.

\section{Resultados}

Las características sociodemográficas de las mujeres estudiadas mostraron diferencias estadísticamente significativas entre los grupos comparados, excepto en cuanto a la raza (cuadro I).

Del total de mujeres que abortaron el primer embarazo, casi $60 \%$ eran menores de 20 años y sólo 6\% tenían 25 y más años, mientras que de las que parieron, las menores de 20 años representaron $44 \%$, y las de 25 y más años, $15 \%$. La raza mostró una distribución similar en los dos grupos, y en ambos predominó la blanca. No se encontraron diferencias estadísticamente significativas para esta variable. De las que abortaron, 55\% tenían escolaridad de preuniversitario, y sólo 5.5\% eran universitarias; de las que parieron, $49.8 \%$ tenían preuniversitario terminado y casi $10 \%$ eran universitarias. Del total de mujeres que abortaron, $56 \%$ eran solteras, mientras que entre las que parieron $70 \%$ estaban casadas y sólo 15\% eran solteras. Casi $60 \%$ de las mujeres que abortaron no tenían empleo remunerado, y entre las que parieron, $64.5 \%$ sí lo tenían. De las que abortaron, 32.7 y $26.7 \%$ eran amas de casa y estudiantes, respectivamente, mientras que más de la mitad $(54.9 \%)$ de las que parieron eran profesionales. Entre las que abortaron, $81.5 \%$ iniciaron sus relaciones sexuales antes de los 20 años y sólo $0.9 \%$ lo hicieron a los 25 años o más de edad, mientras que $77.2 \%$ de las que parieron habían tenido sus primeras relaciones sexuales antes de los 20 años, y 2.3\%, a los 25 y más años.

Se estimaron las RM crudas y ajustadas por edad, en todas las variables que mostraron diferencias significativas $(p<0.05)$ (cuadro II).

Las mujeres de 20-24 años presentaron 2.1 veces más riesgo de abortar el primer embarazo que las de 25 y más años. Ese riesgo se incrementó a 3.3 veces más en las menores de 20 años al compararlas con las de 25 años y más; esa asociación fue estadísticamente significativa. Para la situación conyugal la categoría de referencia fueron las casadas: las mujeres unidas presentaron 2.3 veces más riesgo de acudir al aborto inducido que las casadas, y en las solteras hubo 9.5 veces más riesgo que en las casadas. Al ajustar esta asociación por edad, la RM ajustada fue estadísticamente significativa; sin embargo, para este caso la edad no se consideró como factor confusor. Al estimar los riesgos ajustados de acudir al aborto inducido para las variables escolaridad, ocupación, tipo de actividad y edad de las primeras relaciones sexuales, la variable edad se consideró como confusora; no obstante, estas asociaciones no fueron estadísticamente significativas.

\section{Discusión}

El aborto es el método más practicado mundialmente para resolver embarazos indeseados, y su elevada incidencia constituye un problema de salud en diferentes países; ahí donde se prohibe su práctica, la situación se agrava debido a las complicaciones del aborto en condiciones de riesgo. ${ }^{4,5}$ Cabe añadir que en muchas naciones, las dificultades para obtener información válida sobre la práctica del aborto inducido y las variaciones que se obtienen en las estimaciones según el método utilizado, subestiman la real magnitud del aborto y hacen que las estadísticas reportadas sean poco confiables.

Según los estudios de Henshaw, anualmente ocurren entre 36 y 60 millones de abortos en el mundo, y la mitad son inducidos. ${ }^{6}$ Así, entre los ejemplos de frecuencias de abortos inducidos en algunos países están los siguientes: Hungría reportó en 1987 una tasa de aborto de 38.2 por 1000 mujeres en edad fértil; en Rumania, en 1982, la tasa de abortos inducidos fue de 90.9, y en la antigua Unión Soviética, en 1987, se notificaron 111.9 abortos por cada 1000 mujeres en edad fértil.

Se calcula que 25\% de los abortos ocurren en América Latina. ${ }^{7}$ En 1991 la tasa estimada de abortos inducidos en Brazil fue de 36.5 por cada 1000 mujeres en edad fértil; en Colombia, en 1989, de 33.7; en Chile, en 1990, de 45.4; en México, en 1990, de 23.3, y en Perú, en 1989, de 51.9 por cada 1000 mujeres en edad fértil. $^{8}$

En Cuba, las estadísticas continuas muestran un ritmo ascendente de las tasas de aborto inducido desde finales de los años sesenta hasta finales de los ochenta, y en la actualidad se encuentran entre las tasas más altas en el mundo.

En 1970 se realizaron en ese país 36.1 abortos por cada 1000 mujeres en edad fértil; en 1989 fueron 46.8. A partir de ese año aparece una tendencia hacia la disminución, en cifras tanto absolutas como relativas, pero estos datos presentan un cierto sesgo al no incluir las aspiraciones endouterinas para regular la menstruación, realizadas desde 1988. Según algunos estudios, se considera que más de $70 \%$ de esas aspiraciones co- 


\section{Cuadro I

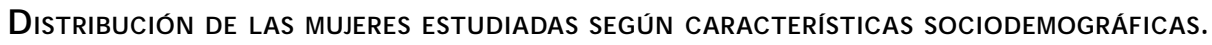 \\ La Habana, Cuba, 1991-1992}

Características

\begin{tabular}{cl} 
& Primer embarazo \\
\hline Aborto & Parto \\
\hline
\end{tabular}

\begin{tabular}{lll}
\multicolumn{2}{c}{ Parto } & \multicolumn{2}{c}{ Total } \\
\cline { 2 - 3 } & $\mathrm{N}$ & $\%$
\end{tabular}

Edad (años)

\begin{tabular}{lrrrrrr} 
Menor de 20 años & 387 & 58.8 & 383 & 44.1 & 770 & 50.4 \\
\hline $20-24$ & 232 & 35.2 & 354 & 40.7 & 586 & 38.3 \\
25 y más & 40 & 6.0 & 132 & 15.2 & 172 & 11.2
\end{tabular}

Raza

Raza

\begin{tabular}{lll} 
Blanca & 405 & 61.4 \\
\hline N egra & 130 & 19.8 \\
\hline Mestiza & 124 & 18.8
\end{tabular}

Escolaridad

\begin{tabular}{lrr} 
Primaria & 10 & 1.5 \\
\hline Secundaria & 249 & 37.8 \\
\hline Preuniversitario & 364 & 55.2 \\
\hline Universidad & 36 & 5.5
\end{tabular}

$\begin{array}{rr}1.5 & 29 \\ 37.8 & 322 \\ 55.2 & 433\end{array}$

\begin{tabular}{rrrr}
29 & 3.3 & 39 & 2.6 \\
\hline 322 & 37.1 & 571 & 37.4 \\
433 & 49.8 & 797 & 52.2 \\
85 & 9.8 & 121 & 7.8
\end{tabular}

Situación conyugal

\begin{tabular}{lllllll} 
Casada & 184 & 28.0 & 598 & 68.8 & 782 & 51.2 \\
\hline Unida & 106 & 16.0 & 145 & 16.6 & 251 & 16.4 \\
\hline Soltera & 369 & 56.0 & 126 & 14.6 & 495 & 32.4
\end{tabular}

0 cupación

\begin{tabular}{lllllll} 
Sí & 267 & 40.5 & 560 & 64.5 & 822 & 53.8 \\
\hline No & 392 & 59.5 & 309 & 35.5 & 706 & 46.2
\end{tabular}

Tipo de actividad

$<0.05$

\begin{tabular}{lll} 
Profesional & 143 & 21.8 \\
\hline O brera & 124 & 18. \\
\hline Estudiante & 176 & 26.7 \\
\hline Ama de casa & 216 & 32.7
\end{tabular}

$\begin{array}{lr}21.8 & 477 \\ 18.8 & \\ 26.7 & 239 \\ 32.7 & 69\end{array}$

$\begin{array}{rr}477 & 54 \\ 84 & 9.7 \\ 69 & 8.0 \\ 239 & 27.4\end{array}$

\begin{tabular}{rrr}
54.9 & 611 & 40.0 \\
\hline 9.7 & 211 & 13.8 \\
8.0 & 250 & 16.4 \\
27.4 & 456 & 29.8
\end{tabular}

Edad de las primeras relaciones sexuales

\begin{tabular}{lrrrrrr} 
Menor de 20 años & 337 & 81.5 & 671 & 77.2 & 1208 & 79.0 \\
\hline $20-24$ & 116 & 17.6 & 178 & 20.5 & 294 & 19.2 \\
\hline 25 y más & 6 & 0.9 & 20 & 2.3 & 26 & 1.8 \\
& & & & & & \\
Total & 659 & 100.0 & 869 & 100.0 & 1528 & 100.0
\end{tabular}

N S: no significativo 
Cuadro II

RAZONES DE MOMIOS CRUDAS Y AJUSTADAS

POR LA EDAD PARA EL EFECTO DE LAS CARACTERÍSTICAS SOCIODEMOGRÁFICAS SOBRE EL ABORTO. La Habana, Cuba, 1991-1992

\begin{tabular}{lcccc} 
Características & $\begin{array}{c}\text { RM } \\
\text { cruda }\end{array}$ & IC & $\begin{array}{c}\text { RM } \\
\text { ajustada }\end{array}$ & IC \\
$\begin{array}{l}\text { Edad (años) } \\
\text { Menor de 20 años }\end{array}$ & 3.33 & $2.27-4.88$ & & \\
\hline $20-24$ & 2.16 & $1.46-3.19$ & & \\
\hline 25 y más & 1.00 & & & \\
& & & & \\
$\begin{array}{l}\text { Escolaridad } \\
\text { Primaria }\end{array}$ & 0.81 & $0.32-1.95$ & 0.35 & $0.15-0.83$ \\
\hline Secundaria & 1.83 & $1.18-2.87$ & 0.83 & $0.51-1.35$ \\
\hline Preuniversitario & 1.98 & $1.29-3.09$ & 1.15 & $0.73-1.81$ \\
\hline Universidad & 1.00 & & &
\end{tabular}

Situación conyugal

\begin{tabular}{llllr} 
Casada & 1.00 & & & \\
\hline Unida & 2.38 & $1.74-3.24$ & 2.19 & $1.61-2.97$ \\
\hline Soltera & 9.52 & $7.27-12.47$ & 9.09 & $6.96-11.86$
\end{tabular}

0 cupación

\begin{tabular}{lllll} 
Sí & 1.00 & & & \\
\hline N o & 2.66 & $1.50-4.74$ & 1.19 & $0.57-2.47$
\end{tabular}

Tipo de actividad

\begin{tabular}{lllll} 
Profesional & 1.00 & & & \\
\hline O brera & 4.89 & $1.89-12.8$ & 2.48 & $0.94-6.52$ \\
\hline Estudiante & 8.35 & $3.24-22.18$ & 1.84 & $0.61-5.55$ \\
\hline Ama de casa & 3.00 & $1.44-6.26$ & 1.02 & $0.43-2.42$
\end{tabular}

Edad de las primeras relaciones sexuales

\begin{tabular}{lllll} 
Menor de 20 años & 2.67 & $1.02-8.17$ & 0.90 & $0.33-2.47$ \\
\hline $20-24$ & 2.17 & $0.81-6.80$ & 1.30 & $0.48-3.51$ \\
\hline 25 y más & 1.00 & & &
\end{tabular}

N ota: las variables que ajustan al modelo de regresión logística son: edad y situación conyugal

rresponden a abortos inducidos, lo cual indica que, en realidad, el aborto ha continuado en niveles altos y que un número considerable de mujeres recurren a esta práctica para regular su fecundidad. ${ }^{*, 9}$

\footnotetext{
* Diagnóstico histológico de embarazo en muestras de material obtenido por aspiración endouterina para regular la menstruación, durante 1986 a 1989. Estadísticas del Hospital Gineco-Obstétrico Eusebio Hernández. La Habana, 1990. Documento no publicado.
}

Una gran proporción de las mujeres cubanas que abortan son adolescentes que están iniciando su vida reproductiva y que aún estudian y no se han casado, como señala este trabajo al identificar como factores de riesgo del aborto inducido del primer embarazo: la edad menor de 24 años (aunque el riesgo es mayor en las menores de 20 años), y estar unidas o solteras.

El perfil sociodemográfico identificado en este estudio denota fallas en la prevención de embarazos indeseados entre las mujeres muy jóvenes y que aún no han cumplido sus expectativas profesionales, laborales y relacionadas con el matrimonio. Estas razones parecen ser incompatibles con la maternidad en el grupo de mujeres estudiadas, las cuales, al contar con un fácil acceso a servicios seguros de aborto, recurren a esta práctica para regular su fecundidad. A pesar de que no se cuenta con estudios comparativos, se esperaría encontrar que las mujeres muy jóvenes decididas a continuar con el embarazo tienen aspiraciones menores que aquellas que deciden abortar. ${ }^{10}$

El aborto en Cuba no es un fenómeno aislado sino la expresión de un problema más complejo determinado por factores que van desde los más generales, relacionados con el medio en que se desenvuelve la mujer, como es la accesibilidad a servicios seguros de aborto inducido, hasta los más específicos como son las características sociodemográficas, las actitudes, los ideales reproductivos y la anticoncepción.

Estudios descriptivos realizados en Cuba confirman lo encontrado en este trabajo, al señalar mayores prevalencias de aborto inducido en las adolescentes, las solteras y las estudiantes. ${ }^{*}$ Pérez Santos analizó 75 expedientes clínicos de mujeres que solicitaron aborto inducido tardío de su embarazo en un hospital de la ciudad de La Habana y encontró que más de 60\% eran menores de 24 años y solteras. ${ }^{11}$ Otro estudio que evaluó la eficacia del misoprostol para interrumpir embarazos en el primer trimestre entre 141 mujeres notificó que el promedio de edad era de 23 años y que $52 \%$ eran solteras. ${ }^{12}$

En la bibliografía consultada se encontró que en países donde el aborto inducido está legalizado y se ofrecen servicios seguros para su práctica, las mujeres que lo solicitan presentan un perfil sociodemográfico muy parecido al de las cubanas.

Más de 50\% de los abortos inducidos en Estados Unidos de América (EUA) ocurren en mujeres sin hijos, $25 \%$ en las adolescentes y más de $80 \%$ en las

\footnotetext{
* El aborto en Cuba. Resumen de publicaciones. La Habana: Dirección Nacional de Estadística, Ministerio de Salud Pública, 1990. Documento no publicado.
} 
solteras. ${ }^{13}$ Según el Centro para la Detección y Prevención de Enfermedades (CDC, por sus siglas en inglés) de Atlanta, en los últimos años en EUA, la tasa de aborto inducido ha declinado en los diferentes grupos de mujeres en edad fértil, pero continúa siendo elevada en las mujeres menores de 25 años y solteras. ${ }^{14}$ En un estudio realizado en 1979 en Hawai se concluyó que las características personales de las mujeres que solicitaban se les practicara su primer aborto eran, predominantemente, tener menos de 25 años, ser solteras y contar con un alto nivel de preparación. ${ }^{15}$

En la mayoría de los países latinoamericanos, donde el aborto es considerablemente restringido, la mujer típica que recibe tratamiento por complicaciones del aborto generalmente tiene entre 20 y 34 años de edad y es madre de dos a tres hijos.

Villarreal y Mora señalan como factores asociados al aborto inducido en Colombia tener entre 20 y 29 años, ser trabajadoras y contar con un nivel de preparación elevado. ${ }^{16}$

En el caso de México, las características de la mujer que aborta de manera inducida, de acuerdo con los estudios de Ordóñez, son: ser multípara, tener entre 25 y 35 años, ser ama de casa, con escolaridad por debajo del promedio y vivir en unión estable. El $64.8 \%$ dio como razones para abortar disponer de un ingreso insuficiente y tener muchos hijos. ${ }^{17}$ Otro estudio realizado en el Hospital General, en la Ciudad de México, con 300 mujeres hospitalizadas por complicaciones del aborto, notifica que sólo $28 \%$ eran menores de 20 años, y $68 \%$ solteras o que vivían en unión libre. ${ }^{18}$

En un estudio que se llevó a cabo en dos hospitales de Santo Domingo, en la República Dominicana, con 350 mujeres que presentaron complicaciones del aborto, se informó que la edad promedio era de 26 años y que solamente $16 \%$ eran adolescentes y $13 \%$ estaban casadas. ${ }^{19}$

\section{Conclusiones}

- El aborto inducido en Cuba es elevado.

- Se identificaron como factores de riesgo asociados con el aborto inducido del primer embarazo en el municipio estudiado: la edad menor de 24 años (aunque el riesgo se incrementa en las menores de 20 años), y estar unidas o solteras.

- Ser muy joven y no haber cumplido con las expectativas profesionales, laborales y relacionadas con el matrimonio parecen ser razones incompatibles con la maternidad en el grupo de mujeres estudiadas.
Por estos motivos, el Programa de Planificación Familiar del país debe brindar una atención preferencial a las mujeres identificadas en el estudio como de alto riesgo de recurrir a la práctica del aborto inducido. Asimismo, se recomienda:

- Ampliar la información dirigida a los varones sobre el uso de métodos anticonceptivos, así como difundir información más objetiva sobre el aborto, para estimular la responsabilidad masculina frente a esta práctica.

- Profundizar en el estudio de la continuidad en el uso de métodos anticonceptivos, de su efectividad y del papel del hombre en la planificación familiar.

\section{Referencias}

1. Dirección Nacional de Estadística, Informe Anual 1993. Estadísticas de Salud del Ministerio de Salud Pública. La Habana: Dirección General de Estadística, Ministerio de Salud Pública, 1993.

2.United N ations. W orld population prospects (The 1992 revision). N ueva York, 1993.

3. Alvarez-Lajonchere C. Commentary on abortion law and practice in Cuba. Int J Gynecol 0 bstet 1989; suppl 3:93-95.

4. Linsking LS. Complications of abortion in developing countries. Popul Rep 1980; Serie F(7):106.

5.T ietze C . Induced abortion. 3a ed. N ueva York: Popul C ouncil, 1979:108. 6. Henshaw SK. Induced abortion: A world review, 1990. Family Plann Perspect 1990;22(2):76-89.

7. Rochat, RW. Induced abortion and health problems in developing countries. Lancet 1980;II(8192):484.

8.The Alan Guttmacher Institute. C landestine abortion:A Latin A merican reality. N ueva York:The Alan Guttmacher Institute, 1994.

9. Boletín Estadístico del Ministerio de Salud Pública. La Habana: Dirección General de Estadística, Ministerio de Salud Pública, 1993.

10.Atkin L. El embarazo en la adolescencia en A mérica Latina y el Caribe: causas y consecuencias psicosociales. En:The Pathfinder Fund/The Population Council, ed. Memorias de la Conferencia Internacional sobre Fecundidad Adolescente en América Latina y el Caribe; 1984; 0 axaca, México.

11. Pérez-Santos R.Algunos factores que inciden en la interrupción tardía de la gestación. Rev Cubana 0 bst Ginecol 1988;14(4):64-70.

12. C arbonell JL,Varela L,Velazco A, Fernández C. The use of misoprostol for termination of early pregnancy. Contraception 1997;55:165-168.

13. Henshaw SK. Induced Abortion. A world rewiew. Fam Plann Perspect 1990;22(2):76-89.

14. Koonin LM. Abortion surveillance, United States, 1989. MMW R Morb Mortal W kly Rep 1992;41(5):1-33.

15. Steinhoff P.W omen who obtain repeat abortions: A study based on Record Linkage. Fam Plann Perspect 1979;11(1).

16. Villarreal-Mejía J, Mora-Téllez M. Embarazo indeseado y aborto: determinantes de la interrupción del embarazo no deseado y características de las mujeres que abortan. Bogotá: 0 riéntame, 1992. 
17. O rdoñez BR. Induced abortion in México City: Summary conclusions from two studies conducted by the Mexican Social Security Institute. Epidemiology of abortion and practices of fertility regulation in Latin America: Select reports. W ashington, D.C.: Pan Am Health 0 rganization, Scientific Publication, núm. 306, 1975:26-29.

18. Elu MC. Between debate and suftering:A bortion in Mexico. W ashington, D.C.: UN DP/UN FPA/W HO, Progress in Human Reproduction Research, núm. 29, 1994:5.
19. Paiewonsky D. Social determinants and consequences of induced abortion in the D ominican Republic.W ashington, D.C.:UN DP/UN FDA/W HO, Progress in Human Reproduction Research, núm. 29, 1994:4. 